\title{
Signs of Tops from Highly Mixed Stops
}

\author{
Mihailo Backović \\ Center for Cosmology, Particle Physics and Phenomenology - CP3, Universite Catholique de \\ Louvain, Louvain-la-neuve, Belgium \\ E-mail: mihailo.backovic@uclouvain.be
}

Alberto Mariotti*

Vrije Universiteit Brussel, and International Solvay Institutes, Pleinlaan 2, B-1050 Brussels, Belgium

E-mail: amariotti.vub@gmail.com

\section{Michael Spannowsky}

Particle Physics Phenomenology, Department of Physics, Durham University, Durham DH1 3LE, United Kingdom

E-mail: michael.spannowsky@durham.ac.uk

Supersymmetric extensions of the Standard Model with highly mixed squark flavours beyond minimal flavour violation provide interesting scenarios of new physics, which have so far received limited attention. We present a calculable realization of such scenarios in models of gauge mediation augmented with an extra interaction between the messengers and the up type squark, and we discuss the flavour physics constraints on such models. In a simplified model approach, we explore the new interesting phenomenology of maximal squark flavour mixing at the LHC. The single-top signature can probe squark masses up to $m_{\tilde{u}} \lesssim 950 \mathrm{GeV}$ at LHC14 with $300 \mathrm{fb}^{-1}$. High luminosity LHC14 can reveal the most distinctive signature of highly mixed scenarios, the same sign positive charge di-top.

18th International Conference From the Planck Scale to the Electroweak Scale 25-29 May 2015

Ioannina, Greece

${ }^{*}$ Speaker. 


\section{Introduction}

The first run of the Large Hadron Collider (LHC) has placed stringent bounds on the scale of physics beyond the Standard Model (BSM), putting under pressure most of the candidate scenarios to address the hierarchy problem. In particular, the minimal supersymmetric extension of the Standard Model is at present considerably constrained. However non-standard supersymmetric scenarios, still within the MSSM but with exotic supersymmetric spectra, have already proven to be able to weaken the LHC constraints $[1,2,3,4,5,6]$. With the imminent start of the run 2 of LHC, it is then important to revisit the implementation of supersymmetry and its breaking in the MSSM, and explore the possibility that supersymmetry is realized with a non-minimal pattern in the structure of the soft terms.

As an example of scenarios which were previously less studied, one could consider relaxing the minimal flavour violation (MFV) assumption on SUSY models. One interesting option for non-standard SUSY beyond MFV involves considerations of mixing between squark flavours. Current experimental results, particularly from flavour physics, give strong constraints on mixing between first and second generation squarks, while mixing between third and first/second generations remains relatively unconstrained. Several scenarios of SUSY models with squark mixing have recently been discussed in Refs. [6, 7, 8, 9, 10].

In this note, based on the paper [11], we study the possibility that the low energy part of the supersymmetric spectrum is characterized by a single (right handed) light squark, highly mixed in flavour between stop and up-squark or stop and scharm-squark. We first propose an explicit realization of such scenario in an extension of gauge mediation, also discussing the low energy physics constraints. Then we study the phenomenology at LHC14 for the case where the light squark is maximally mixed between top- and up-type squark. We focus on the collider signatures which are peculiar to a scenario with maximal stop-sup mixing, i.e. single top and same sign di-top production. ${ }^{1}$ Our collider analysis represents a concrete proposal to test the mixing property of the light squark in the next LHC runs.

\section{The model formulation}

Low Energy Constraints In the minimal supersymmetric extension of the Standard Model (MSSM), the supersymmetry breaking parameters (the soft terms) can be generic sources of flavour and CP violation. The flavour bounds in the MSSM can be analysed in a model independent way by constraining the structure of the supersymmetry breaking parameters [32]. The up-type squark mass matrix in the superCKM basis can be written as

$$
\mathscr{M}=\tilde{m}^{2}\left(\mathbb{I}_{6 \times 6}+\delta_{u}\right) \quad \delta_{u}=\left(\begin{array}{ll}
\delta_{u}^{L L} & \delta_{u}^{L R} \\
\delta_{u}^{R L} & \delta_{u}^{R R}
\end{array}\right)
$$

where the dimensionless $\delta_{u}$ parameterise the deviations from flavour alignment. We are interested in flavour mixing showing up in the right-right part of the up-type squark mass matrix, since they

\footnotetext{
${ }^{1}$ These signatures have already been investigated in several new physics scenarios, single top in $[12,13,14,15,16$, 17] and same sign tops in [18, 19, 20, 21, 22, 23, 24, 25, 26, 27, 28, 29, 30, 31].
} 
are less constrained by flavour physics [2]. The matrix $\left(\delta_{u}^{R R}\right)_{i j}$ is a $3 \times 3$ matrix which is determined by the soft mass of the up-type squark $\left(m_{\tilde{u}}^{2}\right)_{i j}$. The most relevant constraint on the mass matrix is obtained through the measurement of the $D_{0}-\bar{D}_{0}$ mixing, which bounds the absolute value of the $\left(\delta_{u}^{R R}\right)_{12}$ to be smaller than $0.05[32,33]$. Apart from this bound, low energy observables do not independently constrain $\left(\delta_{u}^{R R}\right)_{13}$ or $\left(\delta_{u}^{R R}\right)_{23}$. Another possibly relevant constraint to keep into account for the 1-3 mixing is given by the neutron electric dipole moment (EDM) [34]. However, the neutron EDM processes also involve L-R mixing, hence they can be relevant only in the combined presence of large 1-3 RR mixing and (diagonal or off diagonal) large LR mixing. In the following we provide a computable model which induces large off diagonal contribution only to the RR up-type squark mass matrix $\left(m_{\tilde{u}}^{2}\right)_{i j}$ in the framework of extension of gauge mediation, and which is compatible with flavour constraints.

Extended Gauge Mediation Gauge mediation (for a review see [35]) is a predictive framework for supersymmetry breaking which is automatically flavour diagonal and can predict the structure of the soft terms as functions of few UV parameters. The principle of gauge mediation is that the SUSY breaking sector is connected to the MSSM only via SM gauge interactions. Gauge mediation can be deformed by considering additional interactions between the SUSY breaking sector and the MSSM.

As we show in this section, scenarios with large squark mixing can be realized by extensions of gauge mediation augmented with extra superpotential interactions among the messengers and some superfield of the MSSM. This class of models has recently been studied in several papers $[36,37,38,39,40,41,42,43,44,45,46,47,48,49,50,51,52]$. An interesting aspect of these deformations is that they are not automatically diagonal in flavour space, and hence they can represent controllable sources of flavour violation, which are normally absent in vanilla gauge mediation.

The model we propose consists of a pair of messengers in the $\overline{5}+5$ that we denote $\left(\phi_{1}, \phi_{2}, \tilde{\phi}_{1}, \tilde{\phi}_{2}\right)$. We assume that the component of the $\overline{5}$ messengers with the same quantum numbers of right handed down quark interact with the up type quarks via the superpotential couplings

$$
\delta W=\lambda \sum_{i=1}^{3} c_{i} U_{i} \phi_{1(\overline{5}, D)} \phi_{2(\overline{5}, D)},
$$

where the index $i$ runs here on the flavour index. Hence the interaction is not diagonal in flavor, and can induce non-trivial flavor mixing, depending on the values of the vector $\vec{c}=\left\{c_{1}, c_{2}, c_{3}\right\}$. We take the normalization $\sqrt{c_{1}^{2}+c_{2}^{2}+c_{3}^{2}}=1$, while $\lambda$ sets the overall size of the deformation. We take all couplings to be real, in order to not introduce sources of CP violation. The superpotential (2.2) adds to the usual MSSM superpotential which includes the Yukawa couplings and the $\mu$ term.

The supersymmetry breaking superpotential for the two pairs of $5+\overline{5}$ messengers is

$$
W_{R \text {-pres }}=M\left(\phi_{1} \tilde{\phi}_{1}+\phi_{2} \tilde{\phi}_{2}\right)+Y\left(\phi_{1} \tilde{\phi}_{2}+\phi_{2} \tilde{\phi}_{1}\right)
$$

with the SUSY breaking spurion $Y=\theta^{2} F$. The messenger scale $M$ sets the energy scale where the soft masses are generated. Note that there is a residual $\mathbb{Z}_{4} R$-symmetry under which the spurion $Y$ has charge 2, with the other charges reported in Table 1. This implies that gaugino masses and also $A$-terms cannot be generated by this sector and by the deformation $\lambda$. The discrete R-symmetry, 


\begin{tabular}{l||c|c|c|c|c|c|c} 
& $Y$ & $\phi_{1}$ & $\tilde{\phi}_{1}$ & $\phi_{2}$ & $\tilde{\phi}_{2}$ & $U, D, E$ & $Q, H_{u}, H_{d}, L$ \\
\hline $\mathbb{Z}_{4}$ R-symmetry & 2 & 2 & 0 & 0 & 2 & 0 & 1
\end{tabular}

Table 1: $\mathbb{Z}_{4} R$-symmetry charge assignment.

together with a messenger $Z_{2}$ symmetry under which $\phi_{i}$ and $\tilde{\phi}_{i}$ are odd, implies that the deformation (2.2) is the only one compatible with these discrete simmetries and with gauge invariance.

The boundary conditions induced at the messenger scale by this SUSY breaking sector includes gauge mediated contributions to the scalar masses and the contribution arising because of the new interaction $\lambda$. The gauge mediation contribution, defining $\Lambda=\frac{F}{M}$, is

$$
m_{\tilde{f}}^{2}=\frac{2}{\left(16 \pi^{2}\right)^{2}} \sum_{r} C_{r}^{\tilde{f}} g_{r}^{4}\left(2 \Lambda^{2} f_{s}\left(\frac{\Lambda}{M}\right)\right)
$$

where $f_{s}(x)$ is the usual minimal gauge mediation function for sfermions (see e.g. [53]) $f_{s}(x)=$ $1+\frac{x^{2}}{36}+O\left(x^{4}\right)$. The contribution to the soft masses induced by the $\lambda$ deformation are

$$
\begin{aligned}
& m_{U_{i} U_{j}}^{2}=\frac{1}{256 \pi^{4}} c_{i j} \lambda^{2} d_{U}\left(\lambda^{2} d_{\phi}-2 \sum_{r=1,3} C_{r} g_{r}^{2}\right) \Lambda^{2}-\frac{d_{U}}{48 \pi^{2}} c_{i j} \lambda^{2} h\left(\frac{\Lambda}{M}\right) \frac{\Lambda^{4}}{M^{2}} \\
& m_{Q_{i j}}^{2}=-\frac{d_{U} \lambda^{2}}{256 \pi^{4}}\left(y^{\dagger} . c . y\right)_{i j} \Lambda^{2} \\
& m_{H_{u}}^{2}=-\frac{3 d_{U} \lambda^{2}}{256 \pi^{4}} \operatorname{Tr}\left(y^{\dagger} . c . y\right) \Lambda^{2}
\end{aligned}
$$

where we defined the matrix $c_{i j}=c_{i} c_{j}, d_{U}=2$ and $d_{\phi}=4, C_{1}=2 / 5, C_{2}=0, C_{3}=4$. The one loop function $h(x)$ is given in [11] and can be expanded as $h(x)=1+\frac{4}{5} x^{2}+O\left(x^{4}\right)$. Note that the up type right squark gets off diagonal contributions whose flavour structure is determined by the matrix $c_{i j}$. In order to induce non vanishing gaugino masses and sizable scalar masses we consider also in addition to the previous SUSY breaking sector another supersymmetry breaking sector inducing general gauge mediation contribution, with different gaugino and scalar SUSY breaking scales, respectively $\Lambda_{G}$ and $\Lambda_{S}$

$$
\begin{aligned}
& M_{g_{r}}=\frac{g_{r}^{2}}{16 \pi^{2}} \Lambda_{G} \\
& m_{\tilde{f}}^{2}=\frac{2}{\left(16 \pi^{2}\right)^{2}} \sum_{r} C_{r}^{\tilde{f}} g_{r}^{4} \Lambda_{S}^{2} .
\end{aligned}
$$

For simplicity we assume that such contribution is induced at the same messenger scale $M$ as above, which hence sets the range of scales of the renormalisation group $(R G)$ flow.

In summary, the total contribution to the soft masses in the complete model is given by adding expressions $(2.4,2.5,2.6)$.

Numerical analysis We explored the parameter space of this model and the implication for flavor observables using the tools SARAH [54, 55], SPheno [56] and SUSYFLAVOR [57]. The flavour observables that we checked, together with the bounds that we applied, were taken from [58] and 

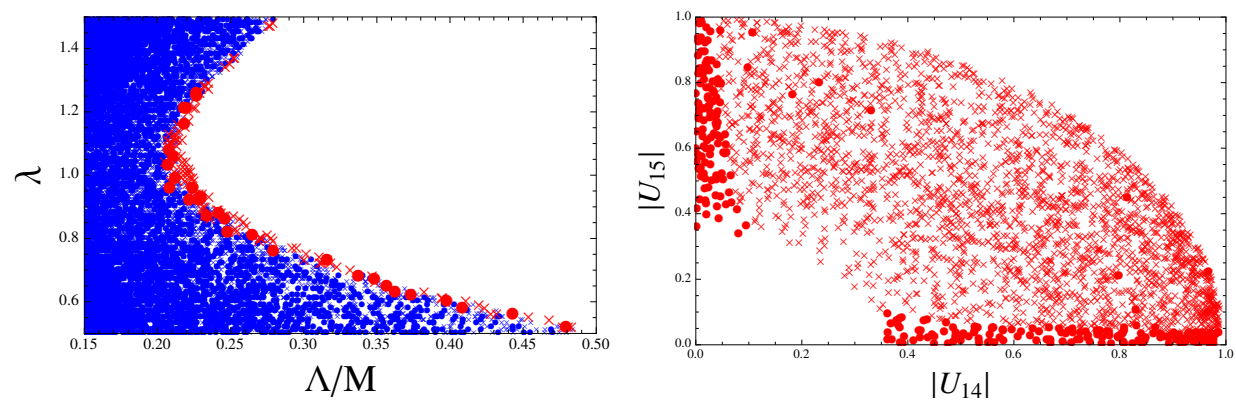

Figure 1: Left: results of the scan on the parameter space of the model on the $(\lambda, \Lambda / M)$ plane. We fixed $\Lambda_{S}=$ $1 \times 10^{6} \mathrm{GeV}, \Lambda_{G}=1.9 \times 10^{5} \mathrm{GeV}$ (corresponding to gluino mass around $1.5 \mathrm{TeV}$ ),$\Lambda=5 \times 10^{5} \mathrm{GeV}$. We scanned over $\lambda, c_{1}, c_{2}, c_{3}$ and $M$. The red points have one squark with mass smaller than $1.5 \mathrm{TeV}$. Right: we focus on a region with light squark: we fixed $\lambda=1$ and we restricted $M$ to be such that $\Lambda / M \simeq 0.2$, varying freely on $\left(c_{1}, c_{2}, c_{3}\right)$; we show only points having one light squark with mass smaller than $1.2 \mathrm{TeV}$, and we plot in the physical mixing angle plane $\left(\left|U_{14}\right|,\left|U_{15}\right|\right)$, where $U_{14}$ is the up component and $U_{15}$ is the charm component of the lightest squark. All points displayed have $m_{h}=125 \pm 2.5 \mathrm{GeV}$.

are listed in details in [11]. The most stringent constraint, as explained above, arises from the $D_{0}$ mixing which impose the bound $\Delta M_{D} \leq 8.82 \times 10^{-15} \mathrm{GeV}$ [59].

We performed a scan by fixing the values of $\Lambda_{G}, \Lambda_{S}$ and $\Lambda$ and varying the messenger mass $M$, the deformation size $\lambda$, and the flavour direction $\vec{c}$ of the deformation. In the left plot of Fig. 1 we show the result in the $(\lambda, \Lambda / M)$ plane. The points respecting flavour constraints are shown as circular dots, while the crosses are points violating flavour observables. The red points are scenarios where the lightest up type squark is lighter than $1.5 \mathrm{TeV}$. The Higgs mass is correct (within the errors) on all the points shown in the plot, due to the large value of $\Lambda_{S}$.

The main effect of the deformation is on the mass squared $m_{U_{i j}}^{2}$ (see eqn. (2.5)), which can be negative (or very small) at the messenger scale and hence positive but small at the EW scale, in the region of moderate $\lambda$ and large ratio $\Lambda / M$. The allowed region in Fig. 1 is determined by the fact that for large values of $\Lambda / M$ the negative one loop contribution to the up-type squark mass (independently on the flavour direction) renders tachionic the squark eigenstate aligned with $\vec{c}$, and the spectrum is rejected. Indeed, the points where there is a single light right-handed up squark with mass smaller than $1.5 \mathrm{TeV}$, the red points, are at the border of the allowed region.

Note that in the red region only one mass eigenstate $\tilde{u}_{1}$ is light and much lighter than all the other squarks. This mass eigenstate will be aligned along the vector $\vec{c}$ in flavor space, as the deformation $\lambda$, and hence it will be a mixture of right handed stop, scharm and up-squark in the combination $^{2}$

$$
\tilde{u}_{1}=U_{14} \tilde{u}_{R}+U_{15} \tilde{c}_{R}+U_{16} \tilde{\tau}_{R} \quad \text { with } \quad\left|U_{14}\right|^{2}+\left|U_{15}\right|^{2}+\left|U_{16}\right|^{2}=1
$$

where $\left|U_{14}\right| \simeq c_{1},\left|U_{15}\right| \simeq c_{2},\left|U_{16}\right| \simeq c_{3}$.

In the plot on the right of Fig. 1 we focus on points with one light up-type squark with a mass smaller than $1.2 \mathrm{TeV}$. The red crosses are points which are not allowed because they do not respect flavour observables, while the circular dots are viable points. Hence in this model we can

\footnotetext{
${ }^{2}$ We used here the SLHA2 notations [60] (i.e. $4 \leftrightarrow \tilde{u}_{R}, 5 \leftrightarrow \tilde{c}_{R}, 6 \leftrightarrow \tilde{t}_{R}$ ).
} 


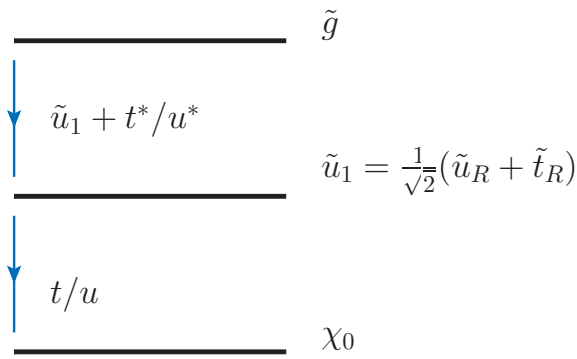

Figure 2: Maximally Mixed Sup-Stop (MMUT) scenario: the simplified model considered in the collider analysis.

realize scenarios where the lightest squark is a highly mixed state in the flavor basis, either supstop or scharm-stop (essentially the circular red points along the two axes). In the plots, all points have a viable Higgs mass, which is a consequence of the fact that at least one of the two stops is very heavy, with a mass set mainly by $\Lambda_{S}$. However the Higgs mass constraint implies that the deformation cannot lower too much the mass of the lightest eigenstate, if this has a significant component in the direction 3 in flavor space. Hence very low mass values for the lightest squark are allowed only if they are not aligned along the third family. This explains the hole of points in the region of small $c_{1}$ and $c_{2}$, where the deformation is mainly aligned along the direction 3 in flavour (note, we have fixed $\lambda=1$ and that $c_{1}^{2}+c_{2}^{2}+c_{3}^{2}=1$ ).

Summarizing, the deformation presented in this section provides a model realization of scenarios with a light squark with a large stop-sup or stop-scharm mixing. In the following we focus on the case of large sup/stop mixing, assuming a small scharm component, consistently with one set of points shown in Fig. 1. The phenomenology of the complementary case with large scharm/stop mixing been recently discussed in [6].

\section{The collider signatures}

Simplified model Based on the previous analysis of the parameter space of the model, we here define a simplified spectra that we investigate in the phenomenological study of the rest of the paper, and we set our benchmark points. The only light supersymmetric states are a highly mixed right handed up-type squark, that we denote with $\tilde{u}_{1}$, with masses varying between 400 and 1000 $\mathrm{GeV}$, and a pure Bino neutralino $\chi_{0}$, with mass varying between 200 and $\left(m_{\tilde{u}_{1}}-200\right)$. The gluino, which is relevant since it participates in the production mechanism of the light squark, and can also be directly produced, is fixed to $2 \mathrm{TeV}$. We focus on the case with maximal stop-sup mixing, i.e. the light squark state is a perfect mixture of sup and stop right-handed squarks

$$
\tilde{u}_{1}=\frac{1}{\sqrt{2}}\left(\tilde{u}_{R}+\tilde{t}_{R}\right) \text {. }
$$

The simplified model, that we dub as Maximally Mixed Sup-Stop (MMUT) scenario, is shown in Fig. 2. ${ }^{3}$ The branching ratio of the possible decays in Fig. 2 depend generically on the mixing

\footnotetext{
${ }^{3}$ Note that the LSP of the model, not shown in Fig. 2, is the gravitino. We assume that the MSSM sparticle decays into gravitino are always suppressed and hence not play any role in colliders. This is consistent quantitatively with the fact that the SUSY breaking scale for the scalars $\left(\Lambda_{S}\right)$ is typically large in the model to generate a viable Higgs mass.
} 

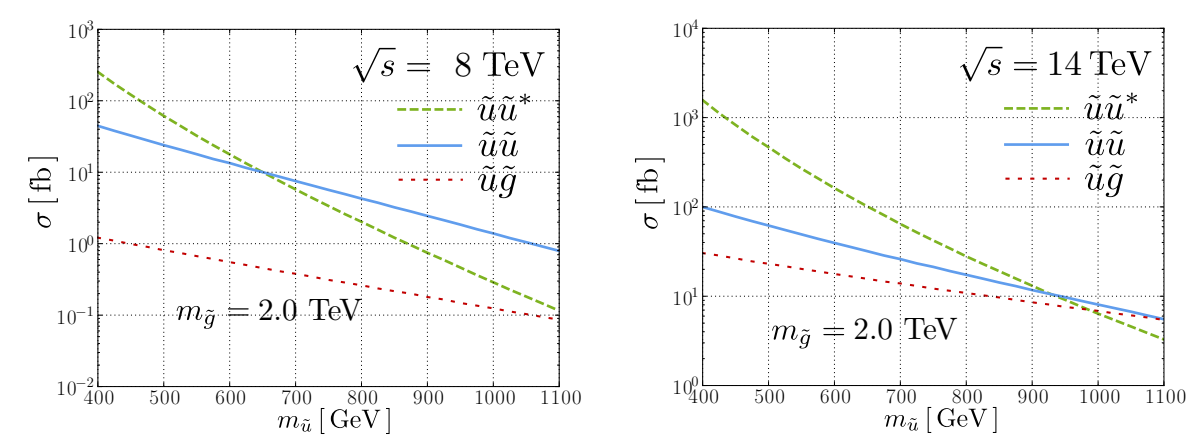

Figure 3: Cross sections in the MMUT model for squark-antisquark, squark-squark and gluino-squark production at LHC8 and LHC14 as a function of the squark mass. The gluino mass is fixed to $2 \mathrm{TeV}$.

angle and can be computed analytically [61, 62] (see [11] for the complete discussion).

We define five benchmark points on which we concentrate in the collider analysis, all with gluino mass fixed at $2 \mathrm{TeV}$, and with different squark and neutralino masses. They are reported in Table 2. The first one has very light squark but quite compressed spectra, with $m_{\tilde{u}_{1}}-m_{\chi_{0}}=250$ $\mathrm{GeV}$. The others have larger squark masses and light or moderately heavy neutralino. By direct

\begin{tabular}{|c|c|c|c|c|}
\hline & $m_{\tilde{g}}$ & $m_{\tilde{u}_{1}}$ & $m_{\chi_{0}}$ & $\tilde{u}_{1}$ mixing angles \\
\hline Benchmark Point 1 & $2 \mathrm{TeV}$ & $450 \mathrm{GeV}$ & $200 \mathrm{GeV}$ & $\left|U_{14}\right|=\left|U_{15}\right|=\frac{1}{\sqrt{2}}$ \\
\hline Benchmark Point 2 & $2 \mathrm{TeV}$ & $700 \mathrm{GeV}$ & $200 \mathrm{GeV}$ & $\left|U_{14}\right|=\left|U_{15}\right|=\frac{1}{\sqrt{2}}$ \\
\hline Benchmark Point 3 & $2 \mathrm{TeV}$ & $700 \mathrm{GeV}$ & $400 \mathrm{GeV}$ & $\left|U_{14}\right|=\left|U_{15}\right|=\frac{1}{\sqrt{2}}$ \\
\hline Benchmark Point 4 & $2 \mathrm{TeV}$ & $950 \mathrm{GeV}$ & $200 \mathrm{GeV}$ & $\left|U_{14}\right|=\left|U_{15}\right|=\frac{1}{\sqrt{2}}$ \\
\hline Benchmark Point 5 & $2 \mathrm{TeV}$ & $950 \mathrm{GeV}$ & $400 \mathrm{GeV}$ & $\left|U_{14}\right|=\left|U_{15}\right|=\frac{1}{\sqrt{2}}$ \\
\hline
\end{tabular}

Table 2: Benchmark points considered in the collider study of the simplified MMUT model.

inspection with CheckMate [63], we verified that the five benchmark points for the MMUT model we defined in Table 2 are still allowed by existing LHC 8 searches.

SUSY production modes Given the simplified spectrum of the MMUT scenario in Fig. 2, at the LHC we expect the following production modes:

$$
p p \rightarrow \tilde{u}_{1} \tilde{u}_{1}^{*} \quad, \quad p p \rightarrow \tilde{u}_{1} \tilde{u}_{1} \quad, \quad p p \rightarrow \tilde{u}_{1} \tilde{g}
$$

Cross sections for squark-antisquark, squark-squark and gluino-squark production at LHC8 and LHC14 are shown in Fig. 3, computed at LO using MadGraph 5 [64]. Note that gluino pair production, gluino-antisquark production and antisquark pair production do not contribute significantly to the SUSY production modes, because of the PDF suppressions. We stress that the production modes of squark-squark and gluino-squark are sizeable only because of the up component in the lightest squark, and thus they are weighted by the mixing angle.

One can generate the following signals from squark-antisquark pair production

$$
p p \rightarrow \tilde{u}_{1} \tilde{u}_{1}^{*} \rightarrow\left(j j \chi_{0} \chi_{0}, j t \chi_{0} \chi_{0}, t t^{*} \chi_{0} \chi_{0}\right)
$$


the following final states from squark-squark pair production

$$
p p \rightarrow \tilde{u}_{1} \tilde{u}_{1} \rightarrow\left(j j \chi_{0} \chi_{0},, j t \chi_{0} \chi_{0}, t t \chi_{0} \chi_{0}\right)
$$

and the following signatures from squark-gluino associated production

$$
p p \rightarrow \tilde{u}_{1} \tilde{g} \rightarrow\left(j j j \chi_{0} \chi_{0}, j j t \chi_{0} \chi_{0}, j j t^{*} \chi_{0} \chi_{0}, j t t^{*} \chi_{0} \chi_{0}, j t t \chi_{0} \chi_{0}, t t t^{*} \chi_{0} \chi_{0}\right)
$$

Besides the usual supersymmetric signatures of jets plus $\mathbb{E}_{T}$ or of top-antitop pair plus $\mathbb{E}_{T}$, among the possible final state we can find the single top (hence single lepton) and same sign tops (hence two positive same sign leptons). Especially this second signature, i.e. same sign tops, is a unique consequence of the maximal flavour mixing in our MMUT scenario, and can be considered as a probe of the mixing angle of the lightest squark state. In the next section we will study the prospects for these two new channels at LHC14 for the five benchmark points of the MMUT simplified model.

\subsection{Top signatures at $\mathrm{LHC14}$}

Event Generation and Reconstruction We generate all events using LO MadGraph 5 [64] with the NNPDF 2.3 [65] set for the parton distribution functions. Upon hard level process generation, we further shower the events using Pythia 6 [66]. We match the background event samples, where relevant, to extra jets using the MLM matching scheme [67]. Our analysis includes detector effects on event reconstruction, where we utilise Delphes 3 [68] with the default CMS settings for event reconstruction, $b$-tagging efficiencies and lepton isolation.

In the following we consider signatures of our model in the single top (i.e. $l+\mathbb{E}_{T}+b$ ) channel as well as the same sign positive top (i.e. $l^{+} l^{+}+\mathbb{E}_{T}$ ) channel. For single-top final state we consider as SM background $t \bar{t}$ and $W+b \bar{b}$ events matched to one extra jet as well as $t W$ events, where we require at least one lepton of unspecified charge at hard process generation level.

Our SM background samples for the same sign lepton analysis consist of $t \bar{t}$ and $W+b \bar{b}$ events matched to one extra jet, where we require one positively charged lepton at generator level. In addition, we also consider rare SM processes where we include production of $t \bar{t} W, t \bar{t} Z, Z Z, W^{+} W^{+} W^{-}$ and $W Z$. Here we require at least one positive lepton in the final state.

In order to improve our estimates of background event yields, we normalise the $t \bar{t}$ production cross sections to the NNLO+NNLL value of Ref. [69], while we assume a conservative K-factor of 1.4 for $W+b \bar{b}$ and single top production and 1.3 for rare SM processes. For the signal generation, we always assume a K-factor of 1.1 for $\tilde{u} \tilde{u}, 1.4$ for $\tilde{u} \tilde{g}$ and 1.5 for $\tilde{u} \tilde{u}^{*}$ production $[70,71]$.

Single-top Channel Our analysis of the single top channel $\left(i . e . l+\mathbb{E}_{T}+b\right)$ is inspired by previous work of Ref. [72]. We include modifications to optimise the analysis for the high luminosity LHC. Besides minimal cuts on the final state object identification, we apply $\mathbb{E}_{T}>250 \mathrm{GeV}$ and we choose $m_{T}>(210,310,410) \mathrm{GeV}$ for the benchmarks with $m_{\tilde{u}}=(450,700,950) \mathrm{GeV}$ respectively.

Our results, summarised in Table 3 , show that the $m_{\tilde{u}}=450 \mathrm{GeV}, m_{\tilde{\chi}_{0}}=200 \mathrm{GeV}$ benchmark point is discoverable with the signal significance of $S / \sqrt{B} \gg 5$ with $L=300 \mathrm{fb}^{-1}$ of integrated luminosity at LHC Run II, while with the same amount of data we should be able to rule out our model for squark masses of roughly $\lesssim 1 \mathrm{TeV}$

A potential discovery of a signal in the $l+E_{T}+b$ channel would give indirect evidence for the existence of supersymmetry, but would not provide information on the degree (if any) of the 


\begin{tabular}{c|ccc}
$m_{\tilde{\chi}_{0}} / m_{\tilde{u}}(\mathrm{GeV})$ & 450 & 700 & 950 \\
\hline 200 & $(0.32,20.0)$ & $(0.24,11.0)$ & $(0.12,3.8)$ \\
400 & - & $(0.11,5.3)$ & $(0.11,3.4)$
\end{tabular}

Table 3: Summary of reach for benchmark points in the single top channel. The table entries show $S / B$ and $S / \sqrt{B}$ at $300 \mathrm{fb}^{-1}$ respectively which can be achieved at LHC Run II in the single top channel. The masses of squarks and neutralinos are listed on in the topmost row and leftmost column respectively. All results assume $m_{\tilde{g}}=2 \mathrm{TeV}$.

$\tilde{u}-\tilde{t}$ mixing, as even then minimal flavor-conserving SUSY models predict signals in the singlelepton channel. Measuring additional channels would be required to determine the presence of $\tilde{u}-\tilde{t}$ mixing, of which we find that the channel with two positively charged leptons is an excellent candidate.

Same sign top Channel Within the framework of SUSY, the "smoking gun" signal of the maximally mixed $\tilde{u}$ model at the LHC is the final state containing two positive leptons and large missing energy.

The same-sign positive lepton final states are a consequence of the $u u \rightarrow \tilde{u} \tilde{u}$ process, with consecutive decays to $t \chi_{0}$, or of the $u g \rightarrow \tilde{u} \tilde{g}$ process, where $\tilde{u}$ decay to $t \chi_{0}$ and the decay chain of the gluino present one positive top. Though suppressed by two powers of the $\tilde{u}-\tilde{t}$ mixing angle, as well as the small branching ratios of $W$ to leptons, the $l^{+} l^{+}+E_{T}$ channel offers a very clean probe of the presence of large $\tilde{u}-\tilde{t}$ mixing. Production of $\tilde{u}^{*} \tilde{u}^{*}$ and $\tilde{g} \tilde{u}^{*}$ which would yield two negative sign leptons in the final state, contributes only few $\%$ to the total signal cross section, due to the PDF suppressions. In the context of SUSY, the strong PDF suppression is a valuable feature of signals with large $\tilde{u} \tilde{t}$ mixing, as other RP conserving supersymmetric models can predict samesign lepton signals with the same amounts of $l^{+} l^{+}$and $l^{-} l^{-}$events [31]. Furthermore typical RPV models with Baryon violation lead to signals with dominant $l^{-} l^{-}$[27], while RPV models which also includes lepton number violation could lead to dominant $l^{+} l^{+}$[29].

Fig. 4 shows the characteristic kinematic distributions of the signal and background in the $l^{+} l^{+}+\mathbb{E}_{T}$ channel. SM backgrounds consist mainly of $\mathscr{O}(10)$ fb level rare SM processes (here we consider $t \bar{t} W, t \bar{t} Z, Z Z, W^{+} W^{+} W^{-}$and $W^{+} Z$ ), as well as SM $t \bar{t}$ and $W+b \bar{b}$, where the same sign lepton background comes mainly from leptonic $b$ decays which yield isolated leptons. With the exception of rare processes, the probability that SM processes contain two positive isolated leptons is tiny, yet significant due to the large production cross sections. In addition, both the amount of missing energy and the transverse mass of the signal events are much larger than in the SM backgrounds, as shown in the right panel of Fig. 4.

In order to maximise the signal significance in the same-sign positive lepton channel, here we employ a set of cuts:

$$
\begin{array}{cc}
N_{l^{+}}\left(p_{T}>20 \mathrm{GeV}, \eta<2.5\right)=2, & E_{T}>120 \mathrm{GeV}, \\
N_{j}\left(p_{T}>30 \mathrm{GeV}, \eta<2.5\right)>1, & m_{T}\left(l_{1}^{+}\right)>m_{T}^{\min }, \\
N_{l^{-}}\left(p_{T}>20 \mathrm{GeV}, \eta<2.5\right)=0 &
\end{array}
$$

where $l^{+-}$refer to isolated positive/negative leptons and $l_{1}^{+}$refers to the highest $p_{T}$ positive lepton in the event. For the purpose of illustration, we set $m_{T}^{\min }=(200,300,400) \mathrm{GeV}$, for $m_{\tilde{u}}=$ 

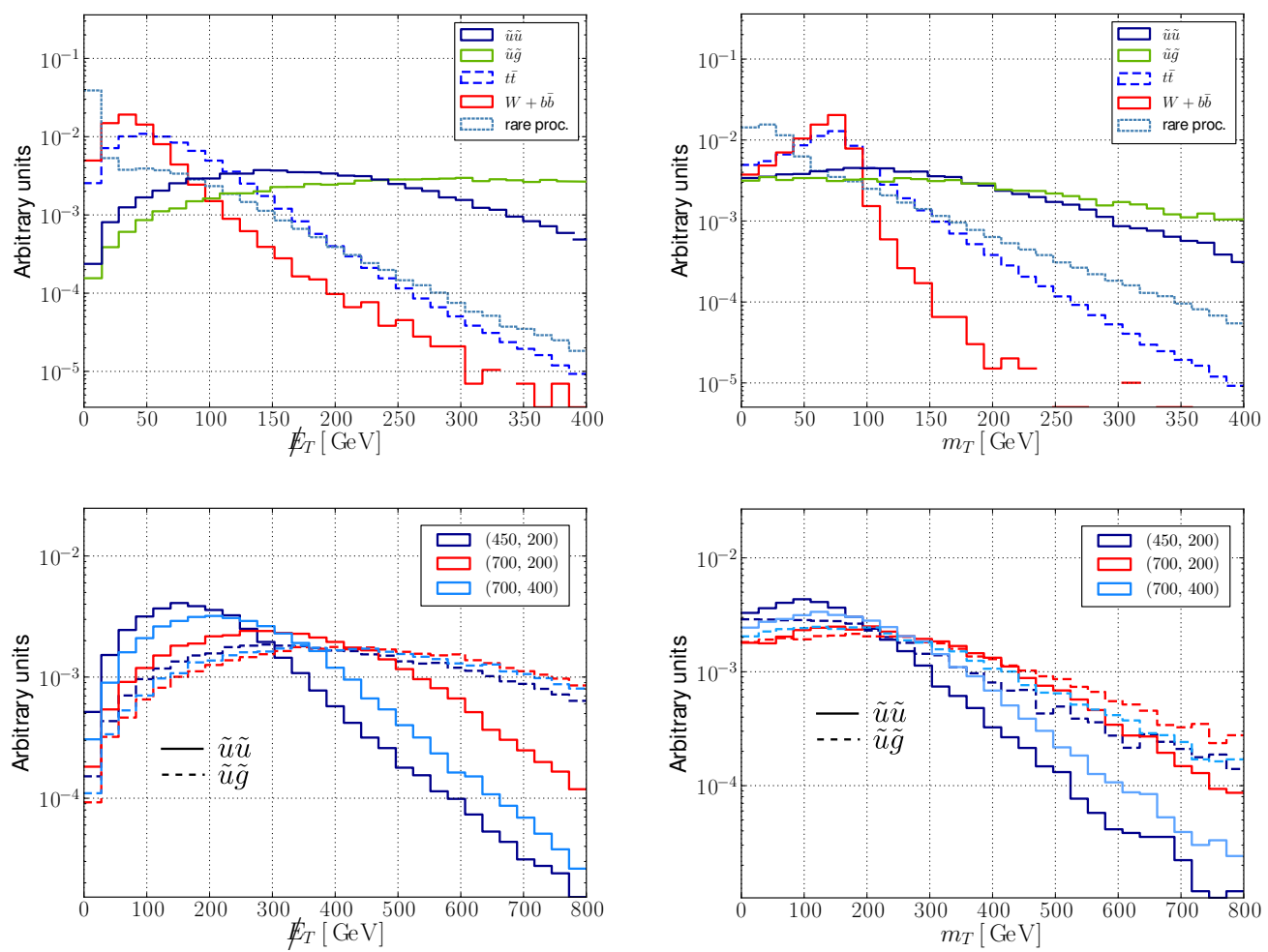

Figure 4: Kinematic distributions relevant for the signal search in the $l^{+} l^{+}+\mathbb{E}_{T}$ channel. All histograms are normalised to unit area. The label "rare proc." includes SM production of $t \bar{t} W, t \bar{t} Z, Z Z, W^{+} W^{+} W^{-}$and $W Z$. In the top panels, we are showing only the benchmark point with $\left(m_{\tilde{u}}, m_{\tilde{\chi}_{0}}\right)=(450,200) \mathrm{GeV}$. In the bottom panels, the values in the legend represent different $\left(m_{\tilde{u}}, m_{\tilde{\chi}_{0}}\right)$ benchmark points.

$(450,700,950) \mathrm{GeV}$ respectively.

Table 4 shows an example cutflow for the benchmark parameter point with $m_{\tilde{u}}=450 \mathrm{GeV}$. The requirement on the lepton multiplicity efficiently reduces the $t \bar{t}$ and $W+b \bar{b}$ backgrounds, while the $E_{T}$ cut efficiently suppresses the rare-process contribution to the total event yield. We find that a minimal set of cuts in Eq. 3.5 results in a factor of $\sim 10-15$ improvement in $S / B$, at a cost of $\sim 50 \%$ in signal efficiency.

\begin{tabular}{l|cc|ccc|c|c}
\multicolumn{7}{c}{$m_{\tilde{u}}=450 \mathrm{GeV}, m_{\tilde{\chi}_{0}}=200 \mathrm{GeV}, m_{\tilde{g}}=2 \mathrm{TeV}$} \\
\hline$l^{+} l^{+}+E_{T}+j j$ & $\tilde{u} \tilde{u}$ & $\tilde{u} \tilde{g}$ & $t \bar{t}$ & $W+$ jets & rare proc. & $S / B$ & $S / \sqrt{B}\left(3000 \mathrm{fb}^{-1}\right)$ \\
\hline$N_{l^{+}}=2, N_{l^{-}}=0$ & 0.21 & 0.037 & 0.067 & 0.022 & 19.0 & 0.013 & 3.1 \\
$N_{j}>1$ & 0.20 & 0.037 & 0.033 & 0.022 & 14.0 & 0.017 & 3.4 \\
$E_{T}>120 \mathrm{GeV}$ & 0.12 & 0.034 & 0.033 & $<0.01$ & 1.2 & 0.12 & 7.6 \\
$m_{T}>200 \mathrm{GeV}$ & 0.080 & 0.019 & $<0.01$ & $<0.01$ & 0.45 & 0.21 & 7.9
\end{tabular}

Table 4: Example cutflow in the $l^{+} l^{+}+\mathbb{E}_{T}$ channel. The entries show cross sections in fb after each consecutive cut. The label "rare proc." includes SM production of $t \bar{t} W, t \bar{t} Z, Z Z, W^{+} W^{+} W^{-}$and $W Z$. We compute $m_{T}$ using the hardest positive lepton in the event and $E_{T}$. 


\begin{tabular}{c|ccc}
$m_{\tilde{\chi}_{0}} / m_{\tilde{u}}(\mathrm{GeV})$ & 450 & 700 & 950 \\
\hline 200 & $(0.21,7.9)$ & $(0.31,7.5)$ & $(0.26,4.1)$ \\
400 & - & $(0.22,5.3)$ & $(0.26,4.0)$
\end{tabular}

Table 5: Summary of reach for benchmark points in the same sign positive lepton channel. The table entries show $S / B$ and $S / \sqrt{B}$ at $3000 \mathrm{fb}^{-1}$ respectively which can be achieved at LHC Run II in the same-sign positive lepton channel. The masses of squarks and neutralinos are listed on in the topmost row and leftmost column respectively. All results assume $m_{\tilde{g}}=2 \mathrm{TeV}$.

Table 5 shows a summary of results on five benchmark points in the $m_{\tilde{u}}, m_{\tilde{\chi}_{0}}$ space. We find that the LHC can achieve a signal significance of $5 \sigma$ for $\tilde{u}$ with masses of up to $\sim 700 \mathrm{GeV}$ for a neutralino mass $\lesssim 400 \mathrm{GeV}$. A significance higher than $3 \sigma$ can be achieved for masses up to $\sim 1 \mathrm{TeV}$, assuming the neutralino masses of $\lesssim 400 \mathrm{GeV}$, suggesting that the same sign positive lepton channel could rule out the model up to $\sim 1 \mathrm{TeV}$ at the high luminosity LHC.

We conclude that the reach of the single top channel at $300 \mathrm{fb}^{-1}$ is comparable to the reach of the same-sign positive leptons search at $3000 \mathrm{fb}^{-1}$. Therefore in case a signal is observed in the single top channel at $300 \mathrm{fb}^{-1}$, the high luminosity LHC should be able to probe and measure possible large squark mixings searching for same sign tops.

\section{Conclusions}

The canonical paradigm of supersymmetry typically assumes aligned or diagonal flavour structure for the soft terrms. Yet, the possibility of large off diagonal entries represents a viable and interesting option to be investigated in the context of the MSSM.

In this note we studied a class of models of supersymmetry with large mixing in the righthanded up-type squark mass matrix in the context of extended gauge mediation. Our analysis includes the study of the constraints imposed on the parameter space by flavour observables, as well as the prospects for LHC Run II to discover models with large squark mixings. We find that the single-top signature can be accessible at LHC14 with $300 \mathrm{fb}^{-1}$. The more distinctive feature of the maximally mixed sup-stop scenario, the same sign positive lepton final state, can be eventually probed at the high luminosity LHC. A combination of the two searches could provide useful insight into the flavor mixing properties of the light squark state.

\section{Acknowledgments}

M.B. would like to thank the University of Kansas phenomenology group for their hospitality during the final stages of the project. M.B. and A.M. are supported in part by the Belgian Federal Science Policy Office through the Interuniversity Attraction Pole P7/37. A. M. is a Pegasus FWO postdoctoral Fellowship. A. M. is also supported in part by the Strategic Research Program High Energy Physics and the Research Council of the Vrije Universiteit Brussel.

\section{References}

[1] G. Bozzi, B. Fuks and M. Klasen, Phys. Rev. D 72, 035016 (2005) [hep-ph/0507073]. 
[2] S. Dittmaier, G. Hiller, T. Plehn and M. Spannowsky, Phys. Rev. D 77, 115001 (2008) [arXiv:0708.0940 [hep-ph]].

[3] B. Fuks, B. Herrmann and M. Klasen, Nucl. Phys. B 810, 266 (2009) [arXiv:0808.1104 [hep-ph]].

[4] T. Hurth and W. Porod, JHEP 0908, 087 (2009) [arXiv:0904.4574 [hep-ph]].

[5] A. Crivellin and J. Girrbach, Phys. Rev. D 81, 076001 (2010) [arXiv:1002.0227 [hep-ph]].

[6] M. Blanke, G. F. Giudice, P. Paradisi, G. Perez and J. Zupan, JHEP 1306 (2013) 022 [arXiv:1302.7232 [hep-ph]].

[7] P. Agrawal and C. Frugiuele, JHEP 1401 (2014) 115 [arXiv:1304.3068 [hep-ph], arXiv:1304.3068].

[8] G. D. Kribs, A. Martin and T. S. Roy, JHEP 0906 (2009) 042 [arXiv:0901.4105 [hep-ph]].

[9] T. Plehn, M. Rauch and M. Spannowsky, Phys. Rev. D 80 (2009) 114027 [arXiv:0906.1803 [hep-ph]].

[10] O. Gedalia, J. F. Kamenik, Z. Ligeti and G. Perez, Phys. Lett. B 714 (2012) 55 [arXiv:1202.5038 [hep-ph]].

[11] M. Backovic, A. Mariotti and M. Spannowsky, JHEP 1506 (2015) 122 [arXiv:1504.00927 [hep-ph]].

[12] A. Arhrib, K. Cheung, C. W. Chiang and T. C. Yuan, Phys. Rev. D 73 (2006) 075015 [hep-ph/0602175].

[13] J. I. Aranda, F. Ramirez-Zavaleta, J. J. Toscano and E. S. Tututi, J. Phys. G 38 (2011) 045006 [arXiv:1007.3326 [hep-ph]].

[14] J. Andrea, B. Fuks and F. Maltoni, Phys. Rev. D 84 (2011) 074025 [arXiv:1106.6199 [hep-ph]].

[15] J. L. Agram, J. Andrea, M. Buttignol, E. Conte and B. Fuks, Phys. Rev. D 89 (2014) 1, 014028 [arXiv:1311.6478 [hep-ph]].

[16] E. Alvarez, E. C. Leskow, J. Drobnak and J. F. Kamenik, Phys. Rev. D 89 (2014) 1, 014016 [arXiv:1310.7600 [hep-ph]].

[17] I. Boucheneb, G. Cacciapaglia, A. Deandrea and B. Fuks, JHEP 1501 (2015) 017 [arXiv:1407.7529 [hep-ph]].

[18] S. Jung, H. Murayama, A. Pierce and J. D. Wells, Phys. Rev. D 81 (2010) 015004 [arXiv:0907.4112 [hep-ph]].

[19] S. K. Gupta, arXiv:1011.4960 [hep-ph].

[20] C. Degrande, J. M. Gerard, C. Grojean, F. Maltoni and G. Servant, Phys. Lett. B 703 (2011) 306 [arXiv:1104.1798 [hep-ph]].

[21] J. Cao, L. Wang, L. Wu and J. M. Yang, Phys. Rev. D 84 (2011) 074001 [arXiv:1101.4456 [hep-ph]].

[22] E. L. Berger, Q. H. Cao, C. R. Chen, C. S. Li and H. Zhang, Phys. Rev. Lett. 106 (2011) 201801 [arXiv:1101.5625 [hep-ph]].

[23] D. Atwood, S. K. Gupta and A. Soni, JHEP 1304 (2013) 035 [arXiv:1301.2250 [hep-ph]].

[24] R. Goldouzian, Phys. Rev. D 91 (2015) 1, 014022 [arXiv:1408.0493 [hep-ph]].

[25] R. Contino and G. Servant, JHEP 0806 (2008) 026 [arXiv:0801.1679 [hep-ph]].

[26] J. Mrazek and A. Wulzer, Phys. Rev. D 81 (2010) 075006 [arXiv:0909.3977 [hep-ph]].

[27] G. Durieux, J. M. Gerard, F. Maltoni and C. Smith, Phys. Lett. B 721 (2013) 82 [arXiv:1210.6598 [hep-ph]]. 
[28] G. Durieux and C. Smith, JHEP 1310 (2013) 068 [arXiv:1307.1355 [hep-ph]].

[29] C. Csaki, E. Kuflik and T. Volansky, Phys. Rev. Lett. 112 (2014) 131801 [arXiv:1309.5957 [hep-ph]].

[30] S. Kraml and A. R. Raklev, Phys. Rev. D 73 (2006) 075002 [hep-ph/0512284].

[31] S. P. Martin, Phys. Rev. D 78 (2008) 055019 [arXiv:0807.2820 [hep-ph]].

[32] W. Altmannshofer, A. J. Buras, S. Gori, P. Paradisi and D. M. Straub, Nucl. Phys. B 830 (2010) 17 [arXiv:0909.1333 [hep-ph]].

[33] M. Ciuchini, E. Franco, D. Guadagnoli, V. Lubicz, M. Pierini, V. Porretti and L. Silvestrini, Phys. Lett. B 655 (2007) 162 [hep-ph/0703204].

[34] A. Dedes, M. Paraskevas, J. Rosiek, K. Suxho and K. Tamvakis, JHEP 1411 (2014) 137 [arXiv:1409.6546 [hep-ph]].

[35] G. F. Giudice and R. Rattazzi, Phys. Rept. 322 (1999) 419 [hep-ph/9801271].

[36] Z. Chacko and E. Ponton, Phys. Rev. D 66 (2002) 095004 [hep-ph/0112190].

[37] A. Delgado, G. F. Giudice and P. Slavich, Phys. Lett. B 653 (2007) 424 [arXiv:0706.3873 [hep-ph]].

[38] G. F. Giudice, H. D. Kim and R. Rattazzi, Phys. Lett. B 660 (2008) 545 [arXiv:0711.4448 [hep-ph]].

[39] A. De Simone, R. Franceschini, G. F. Giudice, D. Pappadopulo and R. Rattazzi, JHEP 1105 (2011) 112 [arXiv:1103.6033 [hep-ph]].

[40] J. L. Evans, M. Ibe and T. T. Yanagida, Phys. Lett. B 705 (2011) 342 [arXiv:1107.3006 [hep-ph]].

[41] Y. Shadmi and P. Z. Szabo, JHEP 1206 (2012) 124 [arXiv:1103.0292 [hep-ph]].

[42] M. Abdullah, I. Galon, Y. Shadmi and Y. Shirman, JHEP 1306 (2013) 057 [arXiv:1209.4904 [hep-ph]].

[43] A. Albaid and K. S. Babu, Phys. Rev. D 88 (2013) 055007 [arXiv:1207.1014 [hep-ph]].

[44] N. Craig, S. Knapen, D. Shih and Y. Zhao, JHEP 1303 (2013) 154 [arXiv:1206.4086 [hep-ph]].

[45] Z. Kang, T. Li, T. Liu, C. Tong and J. M. Yang, Phys. Rev. D 86 (2012) 095020 [arXiv:1203.2336 [hep-ph]].

[46] J. L. Evans, M. Ibe, S. Shirai and T. T. Yanagida, Phys. Rev. D 85 (2012) 095004 [arXiv:1201.2611 [hep-ph]].

[47] J. A. Evans and D. Shih, JHEP 1308 (2013) 093 [arXiv:1303.0228 [hep-ph]].

[48] L. Calibbi, P. Paradisi and R. Ziegler, JHEP 1306 (2013) 052 [arXiv:1304.1453 [hep-ph]].

[49] I. Galon, G. Perez and Y. Shadmi, JHEP 1309 (2013) 117 [arXiv:1306.6631 [hep-ph]].

[50] P. Byakti and T. S. Ray, JHEP 1305 (2013) 055 [arXiv:1301.7605 [hep-ph]].

[51] L. Calibbi, A. Mariotti, C. Petersson and D. Redigolo, JHEP 1409 (2014) 133 [arXiv:1405.4859 [hep-ph]].

[52] J. A. Evans, D. Shih and A. Thalapillil, arXiv:1504.00930 [hep-ph].

[53] S. P. Martin, Phys. Rev. D 55 (1997) 3177 [hep-ph/9608224].

[54] F. Staub, Comput. Phys. Commun. 185 (2014) 1773 [arXiv:1309.7223 [hep-ph]].

[55] W. Porod, F. Staub and A. Vicente, Eur. Phys. J. C 74 (2014) 8, 2992 [arXiv:1405.1434 [hep-ph]]. 
[56] W. Porod and F. Staub, Comput. Phys. Commun. 183 (2012) 2458 [arXiv:1104.1573 [hep-ph]].

[57] A. Crivellin, J. Rosiek, P. H. Chankowski, A. Dedes, S. Jaeger and P. Tanedo, Comput. Phys. Commun. 184 (2013) 1004 [arXiv:1203.5023 [hep-ph]].

[58] http://ckmfitter.in2p3.fr/www/results/plots_moriondl4/num/ckmEval_ results_Moriond14.pdf

[59] Y. Amhis et al. [Heavy Flavor Averaging Group (HFAG) Collaboration], arXiv:1412.7515 [hep-ex].

[60] B. C. Allanach, C. Balazs, G. Belanger, M. Bernhardt, F. Boudjema, D. Choudhury, K. Desch and U. Ellwanger et al., Comput. Phys. Commun. 180 (2009) 8 [arXiv:0801.0045 [hep-ph]].

[61] A. Bartl, W. Majerotto, B. Mosslacher, N. Oshimo and S. Stippel, Phys. Rev. D 43 (1991) 2214.

[62] C. Boehm, A. Djouadi and Y. Mambrini, Phys. Rev. D 61 (2000) 095006 [hep-ph/9907428].

[63] M. Drees, H. Dreiner, D. Schmeier, J. Tattersall and J. S. Kim, Comput. Phys. Commun. 187 (2014) 227 [arXiv:1312.2591 [hep-ph]].

[64] J. Alwall, M. Herquet, F. Maltoni, O. Mattelaer and T. Stelzer, JHEP 1106, 128 (2011) [arXiv:1106.0522 [hep-ph]].

[65] R. D. Ball, V. Bertone, S. Carrazza, C. S. Deans, L. Del Debbio, S. Forte, A. Guffanti and N. P. Hartland et al., Nucl. Phys. B 867, 244 (2013) [arXiv:1207.1303 [hep-ph]].

[66] T. Sjostrand, S. Mrenna and P. Z. Skands, JHEP 0605, 026 (2006) [hep-ph/0603175].

[67] M. L. Mangano, M. Moretti, F. Piccinini and M. Treccani, JHEP 0701, 013 (2007) [hep-ph/0611129].

[68] J. de Favereau et al. [DELPHES 3 Collaboration], JHEP 1402, 057 (2014) [arXiv:1307.6346 [hep-ex]].

[69] M. Czakon, P. Fiedler and A. Mitov, Phys. Rev. Lett. 110, 252004 (2013) [arXiv:1303.6254 [hep-ph]].

[70] D. Goncalves, D. Lopez-Val, K. Mawatari and T. Plehn, Phys. Rev. D 90 (2014) 7, 075007 [arXiv:1407.4302 [hep-ph]].

[71] D. Gonçalves-Netto, D. López-Val, K. Mawatari, T. Plehn and I. Wigmore, Phys. Rev. D 87 (2013) 1, 014002 [arXiv:1211.0286 [hep-ph]].

[72] G. Aad et al. [ATLAS Collaboration], Eur. Phys. J. C 75, no. 2, 79 (2015) [arXiv:1410.5404 [hep-ex]]. 